\title{
DE LA RED DE INFORMACIÓN CONTABLE AGRARIA A LA RED DE SOSTENIBILIDAD DE DATOS AGRARIOS. ORGANIZACIÓN DE LA RECOGIDA DE DATOS EN NAVARRA
}

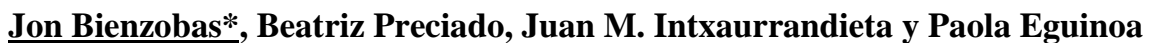

Instituto Navarro de Tecnologías e Infraestructuras Agroalimentarias (Pamplona, jbienzoa@intiasa.es)

\section{Resumen}

INTIA, como entidad de asesoramiento de explotaciones agrarias, participa desde 1989 en la recogida de los datos necesarios para cubrir la muestra que la Red de Información Contable Agraria (RICA) exige a Navarra. Inicialmente, está información era recogida para ofrecer asesoramiento técnico económico a las explotaciones asociadas. En 2005, se empezaron a desarrollar herramientas internas para incorporar indicadores sociales y ambientales a la información económica, con el fin de darle un enfoque global al análisis de la sostenibilidad de los sistemas agrarios. En 2013, INTIA participó en el proyecto FLINT, cuyo objetivo era demostrar la viabilidad de la recogida de información para el desarrollo de indicadores sociales, ambientales y económicos relevantes para la evaluación de políticas públicas.

La estrategia Farm to Fork propone que, a partir de 2022, la actual RICA (FADN) sea sustituida por la Red de Datos sobre la Sostenibilidad Agrícola (RDSA). Por otro lado, la Hacienda Foral ha decidido eliminar el régimen de estimación objetiva (módulos) en IRPF a partir de 2021 obligando a todo agricultor y ganadero a llevar contabilidad.

En esta comunicación se presenta la metodología que propone INTIA para atender las demandas tanto de los agricultores como de la estrategia Farm to Fork y la nueva RDSA (FSDN).

Palabras clave: Indicadores de sostenibilidad, FADN, FSDN, Farm to Fork.

\section{Introducción y objetivos}

El Instituto Navarro de Tecnologías e Infraestructuras Agroalimentarias (INTIA) es una sociedad pública cuya misión es la transferencia y la innovación en el sector agroalimentario con el objetivo de mejorar tanto la viabilidad como su sostenibilidad. Para ello, la gestión técnico económica basada en la contabilidad que se realiza desde 1981 ha sido una herramienta fundamental, facilitando tanto la toma de decisiones de las explotaciones agrarias, como la realización de diagnósticos de los distintos sectores, que puede ser útil para al diseño de políticas públicas. Parte de la información económica recopilada a través de estos servicios ha tenido como destino la Red de Información Contable Agraria (RICA), desde 1989.

Sin embargo, para que los sistemas agrarios sean sostenibles, no sólo deben ser viables económicamente, sino que deben garantizar la protección del medio ambiente y la equidad social (FAO, 2014). Desde 2005, INTIA trabaja en el desarrollo de herramientas y procesos para incorporar información social y ambiental a los diagnósticos de las explotaciones agrarias. En esta línea se desarrollaron diversos proyectos entre los que destaca el proyecto FLINT (2013), cuyo objetivo era demostrar la viabilidad de la recogida de información, en el marco de la RICA (FADN, por sus siglas en inglés), para el desarrollo de indicadores sociales, ambientales y económicos relevantes en la evaluación de políticas públicas.

Los objetivos iniciales de la PAC estaban dirigidos casi exclusivamente al desarrollo económico productivo del sector agrario, entre los que destacan conseguir mejoras en la productividad y renta de los agricultores, estabilizar los mercados y garantizar la seguridad del abastecimiento a precios razonables (Comunidad Económica Europea, 1957). La RICA fue creada en 1965 por la necesidad de contar con una herramienta para medir el efecto de las medidas de la PAC en las explotaciones agrarias (Comunidad Económica Europea, 1965).

No obstante, los objetivos iniciales han ido evolucionando, incluyendo aspectos como el cuidado del clima y del medio ambiente y el fortalecimiento del tejido socioeconómico, por la creciente exigencia de la sociedad europea en materia socio-ambiental. En este escenario, la necesidad de adaptación de la herramienta para monitorizar el grado de cumplimiento de estos objetivos parece evidente. La Comisión Europea (CE), a través de la estrategia Farm to Fork, ha decidido sustituir la actual RICA por una nueva Red de Datos sobre la Sostenibilidad Agrícola (RDSA) a partir del año 2022 (CE, 2020).

El objetivo de esta comunicación es dar a conocer algunos de los indicadores de sostenibilidad que se han estudiado en algunos proyectos de INTIA, la viabilidad de la obtención de nuevos datos requeridos por la RDSA (FSDN, por sus siglas en inglés) y la organización del servicio que se propone en INTIA con vistas a satisfacer tanto los nuevos requerimientos fiscales como estadísticos. 


\section{Metodología}

Ante la coyuntura actual de transformación y con la experiencia de INTIA de más de 30 años en la recogida de datos se han analizado los siguientes aspectos:

- Indicadores sociales y ambientales de interés. Revisión de los indicadores empleados en los diferentes proyectos desarrollados, especialmente FLINT.

- Evolución de la información necesaria para el cálculo de indicadores y viabilidad de su recogida.

- Sistema de recogida de datos en Navarra e implicaciones de la nueva regulación fiscal. Propuesta de futuro.

\section{Resultados}

\subsection{Indicadores socio-ambientales de interés}

En 2005, considerando que la información económica no era suficiente para realizar un diagnóstico global de la sostenibilidad de las explotaciones, se comienza a trabajar en proyectos para incorporar indicadores sociales y ambientales al análisis habitual. Se elabora una batería de 20 indicadores (5 económicos, 7 ambientales y 8 sociales) calculados en base a 120 sub-indicadores, con los cuales se realiza un piloto en 25 explotaciones de ovino.

Cuadro 1. Indicadores aplicados a explotaciones de ovino de leche en Navarra

\begin{tabular}{|l|l|l|}
\hline ECONOMIC & SOCIAL & ENVIRONMENTAL \\
\hline Autonomy & Farm ownership & Livestock and territorial base \\
\hline Risk and diversification & Generation of Emplovment & Land management \\
\hline Costs & Quality of life & Nutrient balance \\
\hline Stability & Quality of labour & Effluent management \\
\hline Profitability & Gender indicators & Landscape and Biodiversity \\
\hline & Animal welfare & Energy \\
\hline & Environment valuation & GHG emissions \\
\hline & $\begin{array}{l}\text { Product quality and } \\
\text { closeness to consumers }\end{array}$ \\
\hline
\end{tabular}

Fuente: Intxaurrandieta et al., 2014

En el marco del proyecto FLINT (2013), se realiza un proyecto piloto de recogida de datos sociales y ambientales en 1.000 explotaciones europeas, de las cuales 165 son recogidas por INTIA. En este proyecto se propusieron 33 indicadores, 9 sobre innovación y economía, 17 medioambientales y 7 sociales.

A raíz del proyecto FLINT, la RICA de Navarra decide en 2016 incorporar algunos de estos indicadores para una sub-muestra de 105 explotaciones, por lo que se puede afirmar que la transición hacia la RDSA en Navarra comienza en 2016.

\subsection{Evolución de la información necesaria para el cálculo de indicadores y viabilidad de su recogida}

En los 40 años que han transcurrido desde que en 1981 el ITG del Vacuno S.A. (actualmente integrado en INTIA) diera sus primeros pasos en la recopilación y análisis de los resultados de gestión técnico económica, la adaptación a las nuevas necesidades que han ido surgiendo ha sido continua. Así, de una gestión por márgenes brutos de rebaño, se pasa a la contabilidad de las explotaciones y de ahí a indicadores de sostenibilidad de los modelos agroalimentarios.

Cuadro 2. Fechas y acontecimientos relevantes para la recogida de datos en Navarra

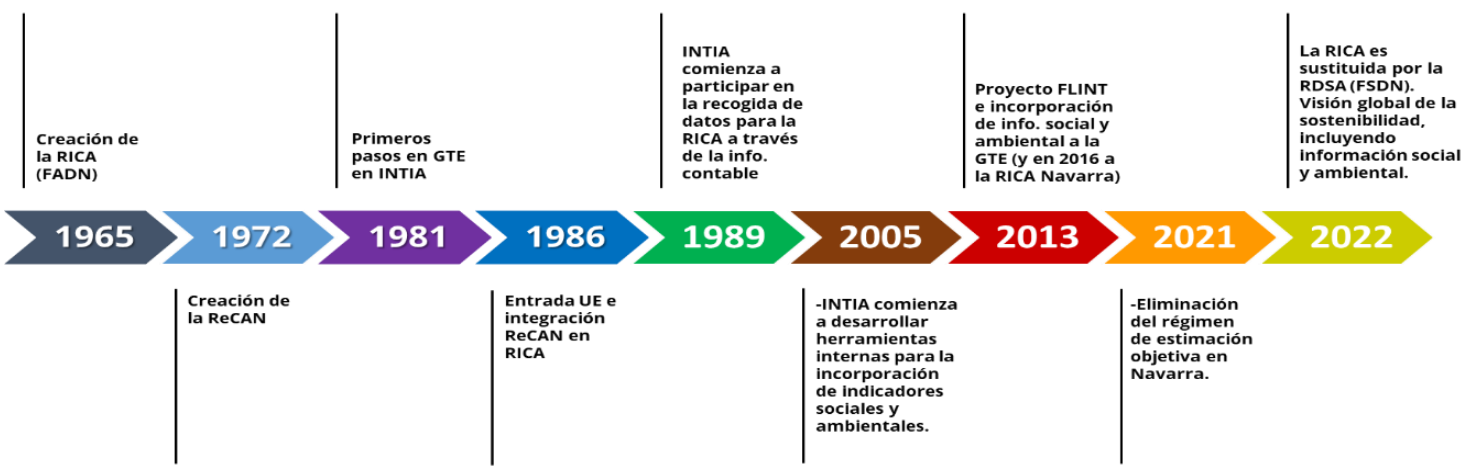

Fuente: Elaboración propia 
Las principales novedades en cuanto a requerimientos de información para el cálculo de indicadores socioambientales han sido:

- Información económico-comercial: modo de comercialización, adscripción a marcas de calidad...

- Información social: Carga de trabajo por tarea, percepción de la calidad de vida y del trabajo, modo de comercialización, género, relevo generacional...

- Información ambiental: biodiversidad, litros de combustibles, kilovatios, piensos y abonos (cantidad y composición) ...

Esta información, añadida a la económica ya utilizada anteriormente, permite el cálculo de indicadores más complejos como las emisiones de gases de efecto invernadero con el objetivo de realizar diagnósticos globales de sostenibilidad de determinadas actividades (Intxaurrandieta et al., 2015).

La viabilidad de la recogida de este tipo de datos ha sido objeto de distintos estudios, el anteriormente mencionado proyecto piloto de Flint en 1.000 explotaciones europeas es el más destacado en esta materia. Con este proyecto, se demuestra que la obtención de estos datos es factible y se considera que la gran clave del éxito es el vínculo de confianza mutuo entre el técnico que recoge la información y el agricultor, algo que no es posible en determinados modelos o sistemas de recogida de datos (Vrolijk et al., 2016).

\subsection{Sistema de recogida de datos en Navarra, nueva regulación fiscal y propuesta de futuro}

En cuanto a las dificultades de recopilar nuevos datos para una muestra aceptable en la RICA, en España se han detectado dos obstáculos fundamentales:

- El modelo de captación de datos para la RICA basado en oficinas contables sin vinculación con centros de investigación o asesoramiento agrario.

- La existencia del régimen fiscal de estimación objetiva (módulos) que implica la no obligatoriedad de la llevanza de libros contables para una gran parte de los agricultores.

En Navarra, la RICA está fundamentada en la información recogida por INTIA, entidad de asesoramiento, lo que facilita el acceso a nuevos datos. Asimismo, la autonomía fiscal de Navarra le ha permitido al Gobierno Foral eliminar el régimen de módulos y obligar a la llevanza de libros contables a la totalidad de los agricultores (Ley Foral 21/2020).

INTIA, ante esta coyuntura está en proceso de organizar un servicio de contabilidad e indicadores que integraría la cumplimentación de las obligaciones contables con una captación de datos sociales y ambientales (unidades físicas) que permitan analizar la sostenibilidad global de las explotaciones. Con esta información, el conocimiento del asesor técnico y otras fuentes de información indirecta disponibles, se minimiza el cuestionario a realizar a los agricultores, uno de los objetivos de la recogida de datos.

En el siguiente cuadro se resumen las fuentes de información de las que se nutre la RICA:

Cuadro 3. Fuentes de información de la RICA en Navarra

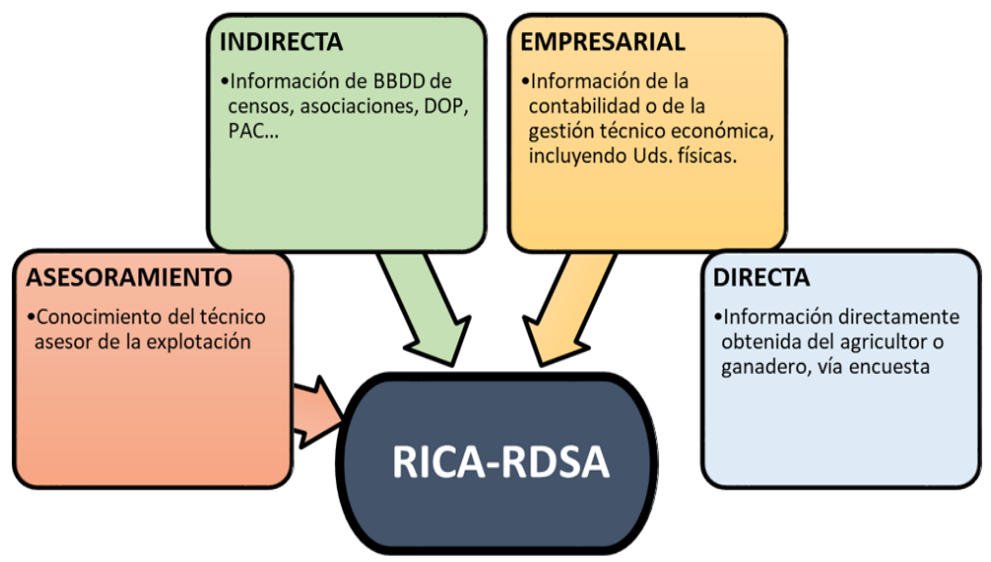

Fuente: Elaboración propia

Con este servicio, el agricultor obtiene:

- Una herramienta y un apoyo para cumplir con sus obligaciones contables y fiscales.

- Asesoramiento técnico, económico y fiscal.

Este servicio permite a INTIA:

- Atender las demandas de los agricultores y proporcionar un servicio orientado a la mejora de la viabilidad de las explotaciones.

- Obtener resultados técnico-económicos y de sostenibilidad de las distintas orientaciones productivas y modelos de producción. 
- Proporcionar la información a la RICA y a la futura RDSA.

\section{Conclusiones}

- La experiencia de INTIA en diferentes proyectos permite avalar la posibilidad de recopilar nuevos datos sociales y ambientales que sirvan para cubrir las necesidades de la nueva RDSA y poder monitorear las nuevas prioridades de la PAC.

- El uso de fuentes indirectas de información y el profundo conocimiento de las explotaciones gracias a los servicios de asesoramiento de INTIA permite disponer de gran volumen de información sin necesidad de nuevos cuestionarios.

- La exigencia de la llevanza de libros contables facilita la disponibilidad de datos y la fidelización de los agricultores en el servicio de contabilidad.

- El desarrollo de herramientas que combinen el cumplimiento de obligaciones fiscales con la recogida de datos extracontables para el análisis de la sostenibilidad reduce tiempos administrativos y costes de recogida.

En definitiva, el escenario actual en Navarra es óptimo para avanzar en la recolección de datos para la nueva FSDN. Tanto el trabajo previo de INTIA en la recogida de datos socio-ambientales como la nueva regulación tributaria que permitirá el acceso a información de explotaciones hasta ahora inaccesibles suponen una gran oportunidad.

\section{Agradecimientos}

Esta comunicación se ha desarrollado en el marco del proyecto EMENSASPI, "Sostenibilidad medioambiental, técnico-económica y social de los sistemas agrarios del Pirineo a través de la cooperación transfronteriza", cofinanciado por el Fondo Europeo de Desarrollo Regional (FEDER).

\section{Bibliografía}

Comunidad Económica Europea (1957). Tratado constitutivo de la Comunidad Económica Europea.

Comunidad Económica Europea (1965). Regulation No 79/65/EEC. Setting up a network for the collection of accountancy data on the incomes and business operation of agricultural holdings in the European Economic Community.

Comisión Europea (2020). De la granja a la mesa. Para un sistema alimentario, justo, saludable y respetuoso con el medio ambiente.

Comunidad Foral de Navarra. Ley 21/2020, de 29 de diciembre, de modificación de diversos impuestos y otras medidas tributarias y de modificación del texto refundido de la Ley Foral de ordenación del territorio y urbanismo. Boletín Oficial de Navarra, de 31 de diciembre de 2020, núm. 304.

FAO (2014). Building a common vision for sustainable food and agriculture. Principles and approaches.

Intxaurrandieta, J.M., Eguinoa, P., Mangado, J.M. (2015). De la huella de carbono a la evaluación multidimensional de la sostenibilidad. Aplicación al ovino de leche en Navarra. AEEA (2015), X Congreso de la Asociación Española de Economía Agraria, Libro de Actas, pp 77-81.

Intxaurrandieta, J.M., Eguinoa, P., Mangado, J.M. (2014). Sustainability indicators in Navarra (Spain). Application to dairy sheep. Pacioli $22^{\text {nd }}$ Workshop. Innovations in farm level data.

Vrolijk, H., Poppe, K., Keszthleyi, Z. (2016). "Collecting sustainability data in different organisational settings of the European Farm Accountancy Data Network". Studies in Agricultural Economics, 118: 138144. http://dx.doi.org/10.7896/j.1626 